\title{
New Methods for Imaging, Isotopic Labeling, and Analyzing Termite Gut Microbes with SEM and NanoSIMS
}

\author{
Kevin J. Carpenter, ${ }^{*}$ Lee Davisson, ${ }^{*}$ Patrick J. Keeling, ${ }^{* *}$ Jennifer Pett-Ridge, ${ }^{*}$ and Peter K.
} Weber*

*Physical and Life Sciences Directorate, Lawrence Livermore National Laboratory, 7000 East Ave, L-231, Livermore, CA 94551, USA

** Botany Department, University of British Columbia, 3529-6270 University Boulevard, Vancouver, BC, V6T 1Z4, CANADA

Microbes inhabiting the guts of phylogenetically "lower" termites and the closely related woodfeeding roach Cryptocercus have attracted the attention of numerous biologists and microscopists for well over 100 years [1]. The combined metabolic activities of hindgut microbes - which include representatives of bacteria, archaea, as well as protists (microbial eukaryotes) - allow their termite hosts to survive on a diet that consists exclusively of wood [2]. The great majority of studies based on microscopy have focused on the protists (comprising mostly parabasalids and oxymonads), not only because of their relatively large size, but also because of the enormous structural complexity and bizarre morphologies present in many species [3, 4]. Additionally, a number of different lines of evidence indicate that the protists (not the bacteria) carry out enzymatic cellulose degradation for the termite host, as well as produce hydrogen gas $[2,3,5]$. Many species of hindgut protists are seen to associate with symbiotic bacteria on their surface, and in cytoplasmic vacuoles [6]; these are thought to provide their protist host (as well as the termite host) with fixed nitrogen in the form of amino acids and other nitrogenous nutrients, while free-swimming spirochetes fix dinitrogen from air [2].

Despite their great structural, evolutionary, and ecological importance, termite hindgut protists — and more recently, their associated symbiotic bacteria - have been studied almost exclusively with light microscopy and TEM (as well as molecular techniques), while studies of morphology with SEM and studies of metabolism using isotopic tracers and NanoSIMS have been rare or nonexistent. Difficulty in fixing whole protist cells from these environments for high vacuum analysis and imaging with high energy electron or ion beams is likely due to their large size (some up to 200 microns long), lack of a rigid cell wall, enormous structural complexity, and the presence of numerous fine-scale structures (some protist species may have tens of thousands of flagella).

Here, we will describe a new approach for SEM and NanoSIMS imaging and analysis of hindgut microbes. The technique relies on two new, simple chemical fixation methods that produce very high quality preservation that retains overall cellular shape (similar to what is observed in light microscopy), fine structures such as flagella (and many newly discovered surface features), as well as membrane integrity of both protists and associated bacteria. Both methods require dilution of hindgut microbes in Trager Medium U buffer [7], and immediate exposure to fixative. The first method uses only osmium tetroxide as a fixative-introduced first as a vapor, followed by direct contact with cells (approximately 1\% v/v). This suspension of fixed cells is then introduced over Millipore Isopore membrane filters (with a 1, 3, or 5 micron pore size) held in Swinnex cartridges affixed to $10 \mathrm{ml}$ syringes. The cells concentrated on filters are then rinsed in buffer and dehydrated with an ethanol series $(50 \%, 70 \%, 90 \%, 100 \%$, and 100\%). This method is quick, does not require an aldehyde fixative, and produces excellent results with most hindgut microbes [4,8]. A second 
method was devised to give improved fixation with more delicate cells. This method introduces unfixed cells in buffer over the Millipore filters (as above), and uses $2.5 \% \mathrm{v} / \mathrm{v}$ glutaraldehyde dissolved in Trager Medium U buffer as a primary fixative, followed by buffer rinses and postfixation in $1 \% \mathrm{v} / \mathrm{v}$ osmium tetroxide. Cells are dehydrated in ethanol (as above). This second method minimizes membrane tearing and cell collapsing, and will also likely extend the useful lifespan of fixed material on stubs [unpubl. data]. Filters with cells fixed by either method are critical point dried in a $\mathrm{CO}_{2}$ critical point dryer with fill and purge rates typically set for twice as long (slow) as normal, and typically manually adjusted and observed over the whole fill and purge sequence to ensure gentle conditions and adequate purging. After drying, the filters are affixed to aluminum SEM stubs with carbon sticky adhesive and coated with $5 \mathrm{~nm}$ of gold or gold-palladium.

Our group has also developed new protocols for ${ }^{15} \mathrm{~N}$ and ${ }^{13} \mathrm{C}$ stable isotope labeling of hindgut microbial communities, followed by NanoSIMS imaging. The NanoSIMS is an imaging mass spectrometer with high $(50 \mathrm{~nm})$ spatial resolution and high sensitivity. These new approaches allow us to visualize the location of metabolic activity (uptake of isotopic tracers) within complex hindgut microbial communities.

For morphology, cells are observed in a cold field emission SEM at $5 \mathrm{kV}$ or lower accelerating voltage to minimize specimen damage and charging. Because many termite gut protist species are very large, it is necessary to use a long working distance ( $25 \mathrm{~mm}$ or greater) to improve depth of field. The use of a low angle secondary electron detector is preferable to minimize charging, as many termite hindgut protist species, particularly those of Parabasalia, have many thousands of flagella which are poorly grounded. These operating conditions are sufficient from the lowest magnification possible up to approximately 10,000x. For imaging at higher magnification, a shorter working distance (e.g., $5 \mathrm{~mm}$ ), lower accelerating voltage, higher $\mathrm{C} 1$ setting (i.e., smaller spot size) and the use of an upper detector, and possibly a smaller objective aperture, is necessary. In the Cameca NanoSIMS 50, fixed gut microbes are interrogated with a $\mathrm{Cs}^{+}$primary ion beam with $50 \mathrm{~nm}$ spatial resolution using L1-2700 and D1-4 aperture. Ratios of ${ }^{13} \mathrm{C} /{ }^{12} \mathrm{C}$ and ${ }^{15} \mathrm{~N} /{ }^{14} \mathrm{~N}$ can be explored with image analyses, which also yield depth profile data.

\section{References}

[1] J. Leidy, Proc. Acad. Nat. Sci. Philadelphia 29 (1877) 146.

[2] M. Ohkuma, Trends in Microbiology 16 (2008) 345.

[3] L.R. Cleveland et al., Mem. Am. Acad. Arts Sci. 17 (1934) 1.

[4] K.J. Carpenter and P.J. Keeling, Journal of Eukaryotic Microbiology 54 (2007) 325.

[5] M.A. Yamin, Science 211 (1981) 58.

[6] R.A. Bloodgood and T.P. Fitzharris, Cytobios 17 (1976) 103.

[7] W. Trager, Biol. Bull. 66 (1934) 182.

[8] K.J. Carpenter et al., Protist 161 (2010) 122.

[9] This research was supported by the Canadian Institute for Advanced Research, the TULA foundation, and the U.S. Department of Energy OBER Genomics:GtL grants (awarded to J. Pett-Ridge and P.K. Weber). The aid of Brian Leander, Elaine Humphrey, Garnet Martens, and Derrick Horne is gratefully acknowledged. 